SCIENCE CHINA

Physics, Mechanics \& Astronomy

\title{
The way to high-performance single nanowire photodetectors: problems and prospects
}

\author{
Jie Mao, Ke Ding, and JianSheng Jie* \\ Institute of Functional Nano \& Soft Materials (FUNSOM), Collaborative Innovation Center of Suzhou Nano Science and Technology \\ (Nano-CIC), Jiangsu Key Laboratory for Carbon-Based Functional Materials \& Devices, Soochow University, Suzhou 215123, China
}

Received October 10, 2016; accepted October 24, 2016; published online November 7, 2016

\begin{abstract}
Citation: J. Mao, K. Ding, and J. S. Jie, The way to high-performance single nanowire photodetectors: problems and prospects, Sci. China-Phys. Mech. Astron. 60, 017031 (2017), doi: 10.1007/s11433-016-0403-3
\end{abstract}

As potential building blocks for micro-nano devices, one-dimensional semiconductor nanowires (NWs) have been widely studied in recent decades. Due to limited dimension and large surface-to-volume ratio, devices based on NWs are more likely to exhibit unique properties. NW photodetectors adopting different materials have been fabricated. However, the performance is greatly affected by several issues such as lattice quality, surface homogeneity, contact electrodes and gas atmosphere. Particularly surface states play a more and more important role. Lots of efforts have been made to remove the obstacles that prevent NWs to be high-performance photodetectors.

As a typical metal oxide semiconductor with wide bandgap, $\mathrm{ZnO}$ NWs are found to be sensitive to ultraviolet (UV) light [1]. However, most $\mathrm{ZnO} \mathrm{NW}$ photodetectors suffer from the persistent photoconductivity (PPC), which means it takes a long time for photocurrent to decrease to the magnitude of dark current after the UV light is switched off. Oxygen vacancies are believed to be the origin of the PPC problem, and this is hard to control during the NW growth process. Prades et al. [2] demonstrated that electron-hole separation near the NW surface has direct impact on the PPC behavior. A larger probing current or a higher oxygen concentration atmosphere would facilitate the access of the electrons to the surface for recombination with the holes, hence reduce the recovery time. This research result shed light on the feasibility of stable and

*Corresponding author (email: jsjie@suda.edu.cn) fast-response photodetectors based on ZnO NWs. Jeon et al. [3] erased the PPC by applying short gate pulses, leading to an oxide semiconductor thin-film transistor array with response time of $25 \mu$ s and frame rate of $150 \mathrm{~Hz}$.

Oxygen adsorption accelerates the vanishing of PPC in n-type $\mathrm{ZnO} \mathrm{NWs}$, while for a p-type system it may bring unexpected results. Zhang et al. [4] found that when p-type $\mathrm{ZnSe}$ :Bi nanowires were exposed to blue light, the photocurrent was smaller than the dark current, which means a negative photoconductivity (NPC). This is attributed to the photodesorption of oxygen molecules. Before illumination, oxygen molecules are adsorbed onto the NW surface, and capture electrons due to the strong electronegativity. When under illumination, photo-generated holes migrate to the NW surface and combine with the captured electrons which results in $\mathrm{O}_{2}$ desorption, while photo-generated electrons left in the core recombine with majority carriers and reduce the conductivity.

In 2015, for the first time another very different NPC phenomenon was observed in narrow band gap n-type InAs NWs by Guo et al. [5]. Nevertheless this NPC is not induced by gas desorption, such NPC is caused by photogating effect. As we known, InAs NWs are regarded as a promising material for broad band detection from UV to infrared (IR) due to narrow bandgap and high electron mobility. However, the abundant surface states and high concentration of background carriers immensely restrict the performance of InAs NWs as photoconductive detectors. If we can take advantage of majority carrier's transport to realize optical detection, the result 
could be amazing. Interestingly, the NW photodetectors designed by Guo et al. are exactly based on this regime. For these InAs NWs showing NPC, surface defects act as a photogating layer trapping hot electrons whereas photogenerated holes remain in the NW core and recombine with free electrons $[5,6]$. These core/shell-like NWs could achieve ultrahigh negative photoconductive gain of $\sim 10^{5}$. However the response speed is relatively slow $(\sim 10 \mathrm{~ms})$. The reason is that the carrier lifetime becomes much longer through the assistance of trap states.

To shorten the response time, it is of great importance to weaken the influence of surface defects. On the basis of the same photogating effect, very recently a visible light-assisted single InAs NW photodetectors with broadband detection from less than $1 \mu \mathrm{m}$ to more than $3 \mu \mathrm{m}$ and fast response speed of tens of microseconds has been demonstrated [7]. This is attributed to that the NW surface defects are not involved in the transportation of infrared light-excited holes and electrons after trapping hot electrons.

However if the number of surface defect states is limited, single InAs NW photodetectors can also exhibit positive photoconductivity like most semiconductors determined by intrinsic photoexcitation [8,9]. Although the surface of this kind NWs does not trap hot electrons like those showing NPC behavior, the electron transport is still sensitive to the ambient environment. Miao et al. [8] found that single InAs NW photodetectors perform better in vacuum than in atmosphere, such as a higher electron mobility and a larger photocurrent. For better performance, surface passivation is a preferable method. The photoresponse time of InAs NWs optimized by surface treatment could be shorter. However the light on/off ratio is still insufficient due to the poor electron-hole separation efficiency and short carrier lifetime.

To enhance the electron-hole separation efficiency, fabricating Schottky contact is a reasonable way. It is found that the responsivity of Schottky-Ohmic contacted single InAs NW photodetectors is almost $300 \%$ larger than that of OhmicOhmic contacted devices [8]. Operating Schottky diodes at a reverse bias voltage is conventional. For the p-type $\mathrm{ZnSe:Bi}$ NW photodetectors discussed in paragraph 3, Zhang et al. [4] used Al (a low work function metal) as one of the electrodes. When applied a reverse bias, these NW photodetectors show positive photoconductivity instead of NPC, indicating that the optical excitation in the Schottky junction area rather than the surface oxygen photodesorption dominates the photocurrent. The high performance of single InAs NW photodetectors mentioned in ref. [7] is also based on the photore- sponse of Schottky junction under reverse bias.

The above discussion illustrates four basic factors that determine the performance of NW photodetectors. In which, gas adsorption can be eradicated by surface passivation, poor light on/off ratio can be enlarged by selective contacts, while NW lattice quality and surface homogeneity need to be meliorated by better growth technology. It is worth noting that Schottky contact is essentially a kind of localized electric field (LEF), and band engineering by LEF is crucial. NW photodetectors based on $\mathrm{p}-\mathrm{n}$ junction and heterojunction also depict nice properties [10]. However the demand for NW growth technology is high. Recently InP and CdS NW photodetectors based on ultrahigh ferroelectric field were fabricated without complicated process $[11,12]$. Due to the full carrier depletion induced by the strong LEF from ferroelectric polarization of $\mathrm{P}(\mathrm{VDF}-\mathrm{TrFE})$, these photodetectors exhibited ultrahigh detectivity. To make clear the distribution of internal electric field, two dimensional laser beam induced current (LBIC) microscopy is a straightforward technical means [13]. According to the photocurrent mapping result, the device structure could be further optimized.

1 H. Kind, H. Yan, B. Messer, M. Law, and P. Yang, Adv. Mater. 14, 158 (2002).

2 J. D. Prades, F. Hernandez-Ramirez, R. Jimenez-Diaz, M. Manzanares, T. Andreu, A. Cirera, A. Romano-Rodriguez, and J. R. Morante, Nanotechnology 19, 465501 (2008).

3 S. Jeon, S. E. Ahn, I. Song, C. J. Kim, U. I. Chung, E. Lee, I. Yoo, A. Nathan, S. Lee, K. Ghaffarzadeh, J. Robertson, and K. Kim, Nat. Mater. 14, 452 (2015).

4 X. Zhang, J. Jie, Z. Wang, C. Wu, L. Wang, Q. Peng, Y. Yu, P. Jiang, and C. Xie, J. Mater. Chem. 21, 6736 (2011).

5 N. Guo, W. Hu, L. Liao, S. P. Yip, J. C. Ho, J. Miao, Z. Zhang, J. Zou, T. Jiang, S. Wu, X. Chen, and W. Lu, Adv. Mater. 26, 8203 (2014).

6 Y. Yang, X. Peng, H. S. Kim, T. Kim, S. Jeon, H. K. Kang, W. Choi, J. Song, Y. J. Doh, and D. Yu, Nano Lett. 15, 5875 (2015), arXiv: 1511.00092 .

7 H. Fang, W. Hu, P. Wang, N. Guo, W. Luo, D. Zheng, F. Gong, M. Luo, H. Tian, X. Zhang, C. Luo, X. Wu, P. Chen, L. Liao, A. Pan, X. Chen, and W. Lu, Nano Lett. 16, 6416 (2016).

8 J. Miao, W. Hu, N. Guo, Z. Lu, X. Zou, L. Liao, S. Shi, P. Chen, Z. Fan, J. C. Ho, T. X. Li, X. S. Chen, and W. Lu, ACS Nano 8, 3628 (2014).

9 Z. Liu, T. Luo, B. Liang, G. Chen, G. Yu, X. Xie, D. Chen, and G. Shen, Nano Res. 6, 775 (2013).

10 J. Miao, W. Hu, N. Guo, Z. Lu, X. Liu, L. Liao, P. Chen, T. Jiang, S. Wu, J. C. Ho, L. Wang, X. Chen, and W. Lu, Small 11, 936 (2015).

11 D. Zheng, J. Wang, W. Hu, L. Liao, H. Fang, N. Guo, P. Wang, F. Gong, X. Wang, Z. Fan, X. Wu, X. Meng, X. Chen, and W. Lu, Nano Lett. 16, 2548 (2016).

12 D. Zheng, H. Fang, P. Wang, W. Luo, F. Gong, J. C. Ho, X. Chen, W. Lu, L. Liao, J. Wang, and W. Hu, Adv. Funct. Mater. in press (2016).

13 W. C. Qiu, and W. D. Hu, Sci. China-Phys. Mech. Astron. 58, 027001 (2015). 